\title{
A rare cadaveric finding of ectopic origin of a bronchial artery: surgical and imaging consequences
}

\author{
K. Natsis ${ }^{1}$, I. Asouhidou'1, 2, V. Vizas ${ }^{1}$, M. Didagelos ${ }^{1}$ \\ ${ }^{1}$ Department of Anatomy, Medical School, Aristotle University of Thessaloniki, Greece \\ 2Department of Cardiac Anesthesia, "G. Papanikolaou" Hospital, Thessaloniki, Greece
}

[Received 27 November 2012; Accepted 27 December 2012]

\begin{abstract}
Bronchial arteries arise normally directly from the descending thoracic aorta, at the level between the fourth and sixth thoracic vertebrae. However, the anatomical variations are very frequent regarding the origin and the number of branches. We present a rare cadaveric finding of an ectopic right bronchial artery originating from the right subclavian artery through a common stem with the right internal mammary artery, and we discuss the clinical significance of this finding. A 72-year-old formalin-embalmed male cadaver was dissected during a routine anatomical course. The right bronchial artery originated from the right subclavian artery with a common stem with the right internal mammary artery. Although ectopic origin of the right bronchial artery from the right internal mammary artery is rare, recognition of this anatomical variation is important in bronchial embolisation due to haemoptysis, in coronary bypass grafting, and in lung transplantation. (Folia Morphol 2013; 72, 1: 78-81)
\end{abstract}

Key words: bronchial artery, subclavian artery, internal mammary/ /thoracic artery, anatomical variation, bypass, lung transplantation

\section{INTRODUCTION}

Bronchial arteries (BA) are small vessels supplying blood to the trachea, bronchi, pulmonary alveolar tissue, bronchopulmonary lymph nodes, pericardium, and oesophagus. The right BA (RBA) normally arises from the third posterior intercostal artery, from the upper left BA (LBA), or directly from the thoracic aorta and runs posterior on the right bronchus when the LBA, usually two, arise directly from the descending thoracic aorta, at the level between the fourth and sixth thoracic vertebra, run posterior to the left bronchus, and are referred to as orthotropic [2]. When the BA originate from the descending aorta at different levels they are referred to as ectopic. The ectopic BA may originate from the aortic arch, abdominal aorta, coronary arteries, subcla- vian or internal mammary arteries (IMA), or the thyrocervical trunk.

Anatomical variations of the BA are very common, with respect to their origin or their number, and being aware of them is important, particularly in the setting of massive haemoptysis, intraarterial infusion of chemotherapeutic agents for lung cancer, or during cardiopulmonary bypass $[1,4]$. Moreover, studies in lung transplantation have shown that reinstitution of BA circulation through anastomosis of the donor's BA to the recipient's bronchial circulation seems to play a significant role [3].

Our study presents a rare cadaveric finding of ectopic origin of the RBA, arising from the right subclavian artery (SA), having a common stem with the

Address for correspondence: I. Asouhidou, MD, PhD, 15-17 Agiou Evgeniou str., Kalamaria 55133, Thessaloniki, Greece, tel: +306944740633, fax: +302310250608, e-mail: iasouhidou@aol.com 


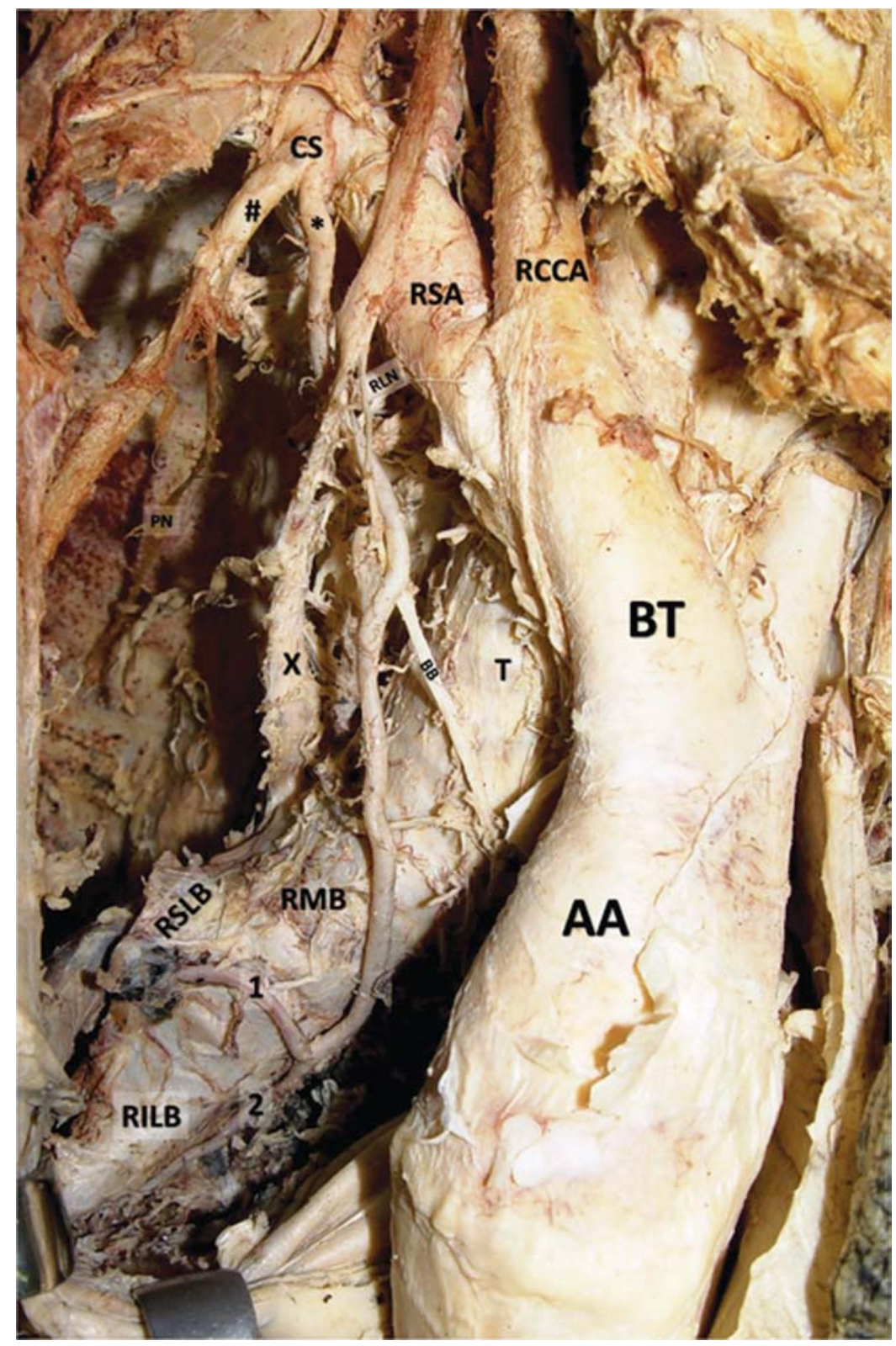

Figure 1. Anterior aspect of the mediastinum after removal of the thoracic cage, preparation of the anatomical structures, and excision of the right lung. The common origin of the right bronchial and right internal mammary artery as well as their course and the nearby anatomical relations are depicted; \#right internal mammary artery; * right bronchial artery; CS - common stem for the right internal mammary artery and the right bronchial artery, RSA — right subclavian artery; RCCA — right common carotid artery; BT — brachiocephalic trunk; AA — aortic arch; PN — phrenic nerve; $\mathrm{X}$ — vagus nerve; RLN — recurrent laryngeal nerve; BB — bronchial (pulmonary) branch of vagus; $\mathrm{T}$ — trachea; RMB — right main bronchus; RSLB — right superior lobar bronchus; RILB — right inferior lobar bronchus; 1 — branch of the right bronchial artery for the RSLB; 2 - branch of the right bronchial artery for the RILB.

right internal mammary artery (RIMA), with further discussion on its clinical impact.

\section{CASE REPORT}

A 72-year-old formalin-embalmed male cadaver was dissected at the Department of Anatomy. After removal of the skin, subcutaneous fat, pectoral muscles, clavicles and anterior surface of the tho- racic cage the heart, lungs, great vessels of the mediastinum, trachea and main bronchi, with all the adjacent anatomical structures were revealed. All findings were recorded and photographed.

The RBA originated from the right SA via a common stem (length $=18 \mathrm{~mm}$, width $=16 \mathrm{~mm}$ ) with the RIMA (Fig. 1). The RBA followed a route behind the vagus nerve at its upper half, passing in front of 
it and in front of the right main bronchus at its lower half, till the lower edge of the right main bronchus. There it formed an almost right angle turning to the right, continuously following the lower edge of the right main bronchus for about $20 \mathrm{~mm}$, before dividing into two branches for the right superior and right inferior lobar bronchi respectively. The internal (lumen) diameter of the ectopic BA was $1.1 \mathrm{~mm}$ (external diameter of $1.5 \mathrm{~mm}$, including arterial wall). There were two LBA arising normally from the thoracic aorta (T5-T6 level). No other anatomical variations were observed.

The cadaver's medical history revealed a large transmural myocardial infarct complicating an episode of angina, causing cardiac arrest and subsequent death of the patient.

\section{DISCUSSION}

Anatomical variations of the origin of BA are very frequent and numerous. The reported prevalence of ectopic BA varies from $8.3 \%$ to $56 \%$ with the majority of them arising from the aortic arch $[1,4,5,7,11]$. According to the literature, the frequency of the ectopic origin is higher for the RBA than for the LBA $[3,4]$.

According to our knowledge there are no photographed data of an ectopic origin of a BA from the IMA in cadaveric specimens. Ectopic origin from the IMA is the least frequent variation. In a study of $184 \mathrm{com}$ puted tomography angiographies (CTA) of the thoracic aorta, Battal et al. [1] found ectopic origin from RIMA in just one of the 432 studied BA. Hartmann et al. [4] studied 214 patients, also by means of CTA, and found only three BA arising from the ipsilateral IMA. Sancho et al. [11] in a series of 300 patients submitted for bronchial embolisation found 25 patients with 27 anomalous $B A$, only one originating from the right SA. Ectopic origin of BA from the SA may be combined with additional vascular anomalies [6].

Ectopic origin seems to play an important role in BA embolisation and management of acute massive haemoptysis. Failure to recognise anatomical variations during angiography of bronchial circulation may induce a partial control of haemoptysis, nontarget embolisation, and unsuccessful outcome $[4,14]$. Therefore, several other BA anastomoses (e.g. the coronary artery) should be sought prior to the administration of embolic agents, in order to prevent potentially catastrophic complications $[8,10]$.

Furthermore, an ectopic BA originating from the IMA also plays a vital role in cardiovascular bypass procedures. RIMA flow to the left anterior descending artery may be seriously compromised due to steal syndrome. This is suspected clinically by myocardial ischaemia of the left anterior wall, electrocardiographic ST segment changes, and unsuccessful weaning from cardiopulmonary bypass. Angiography of the bronchial circulation should be routinely performed in all coronary angiographies to diagnose any anatomical anomaly that compromises left IMA flow. This has recently been enhanced due to strong evidence of the superiority of bilateral use of IMA grafting for patients undergoing bypass regarding single IMA bypass grafting [13].

Suspicion and detection of any possible anatomical variation of BA is significant in lung transplantation since there are centres that routinely re-anastomose the donor's BA that is harvested with the lung to the recipient's BA in order to promote the healing of bronchial anastomosis. The RBA is the main feeding artery of the right main bronchus, the carina, and distal trachea as well as the proximal left main bronchus. It therefore appears that revascularisation of the RBA should, in theory, be sufficient to supply the distal trachea, carina, and proximal bronchial tree, provided the subcarinal and perioesophageal collateral networks are preserved intact [3]. The descending aorta of the donor is posteriorly opened and the ostium of the RBA is identified and isolated within a patch of aorta to facilitate grafting and finally implanted into the ascending aorta of the recipient $[3,9,12]$. In case of ectopic origin of the RBA from the RIMA the plan of surgery has to be modified, especially in cases of single lung transplantation, where collateral network circulation does not exist, and the existence of an ectopic bronchial artery may provoke undesirable problems in an already risky operation.

\section{CONCLUSIONS}

In conclusion, ectopic origin of the RBA from the RIMA is rare, and with no significance in otherwise healthy people, but this anomaly may play a disastrous role in certain clinical circumstances. The presence of BA of anomalous origin should always be considered to prevent a catastrophic outcome.

\section{REFERENCES}

1. Battal B, Akgun V, Karaman B, Bozlar U, Tasar M (2011) Normal anatomical features and variations of bronchial arteries: an analysis with 64Ydetector-row computed tomographic angiography. J Comput Assist Tomogr, 35: 253-259.

2. Cauldwell EW, Siekert RG, Lininger RE, Ansom BJ (1948) The bronchial arteries; an anatomic study of 150 human cadavers. Surg Gynecol Obstet, 86: 395-412. 
3. Couraud L, Baudet E, Nashef SA, Martigne C, Roques X, Velly JF, Laborde N, Dubrez J, Clerc F (1992) Lung transplantation with bronchial revascularisation. Surgical anatomy, operative technique and early results. Eur J Cardiothorac Surg, 6: 490-495.

4. Hartmann IJC, Remy-Jardin M, Menchini L, Teisseire A, Khalil C, Remy J (2007) Ectopic origin of bronchial arteries: assessment with multidetector helical CT angiography. Eur Radiol, 17: 1943-1953.

5. Kasai T, Chiba S (1979) Macroscopic anatomy of the bronchial arteries. Anat Anz, 145: 166-181.

6. Kawate T, Futamata H, Kitami Y, Takeda S (2008) Rare multiple combined anomaly of the vertebral vessels and bronchial artery. Anat Sci Int, 83: 267-272.

7. Lippert H, Pabst R (1985) Arterial variations in man classification and frequency. J.F. Bergmann Verlag, Munchen, pp. 18-19.

8. McPherson S, Routh WD, Nath H, Keller FS (1990) Anomalous origin of bronchial arteries: potential pitfall of embolotherapy for hemoptysis. J Vasc Interv Radiol, 1: 86-88.

9. Nohrgaard MA, Andersen CB, Pettersson G (1998) Does bronchial artery revascularization influence results con- cerning bronchiolitis obliterans syndrome and/or obliterative bronchiolitis after lung transplantation? Eur J Cardiothorac Surg, 14: 311-318.

10. Peynircioglu B, Ergun O, Hazýrolan T, Cil BE, Aytemir K (2007) Bronchial to coronary artery fistulas: an important sign of silent coronary artery disease and potential complication during bronchial artery embolization. Acta Radiol, 48: 171-172.

11. Sancho C, Escalente E, Dominguez J, Vidal J, Lopez E, Valldeperas J, Montana XJ (1998) Embolization of bronchial arteries of anomalous origin. Cardiovasc Intervent Radiol, 21: 300-304.

12. Schreinemakers HHJ. Weder W, Miyoshi S. Harper BD. Shimokawa S, Egan TM, McKnight R, Cooper JD (1990) Direct revascularisation of bronchial arteries for lung transplantation: an anatomical study. Ann Thorac Surg, 49: 44-45.

13. Taggart DP (2012) Bilateral internal mammary arteries: a very important missing trick for coronary artery bypass grafting. Eur J Cardiothorac Surg, 41: 776-777.

14. Yildiz AE, Ariyurek OM, Akpinar E, Peynircioglu B, Cil BE (2011). Multidetector CT of bronchial and non-bronchial systemic arteries. Diagn Interv Radiol, 1: 10-17. 